\title{
EL METROPOLITANO: ¿EN VÍAS DE SER UN MONOPOLIO?
}

THE METROPOLITAN. IN PROGRESS TO BE A MONOPOLY?

Beatriz Herrera García*
Docente Principal de la Facultad de Ciencias Contables
Colaboradores
Alisson Angulo Giraldo-Anaís Arosemena Gómez-Natalie Molina Camone
Myriam Pillaca Rodríguez- Claudia Valdiviezo López
Universidad Nacional Mayor de San Marcos-UNMSM
Lima-Perú

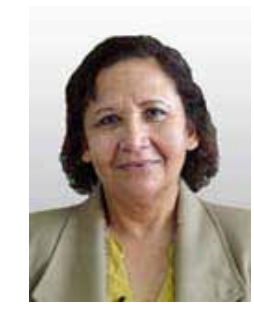

[Recepción: Setiembre de 2012/ Conformidad: Octubre 2012]

\section{RESUMEN}

Actualmente, en nuestra capital, se suscitan problemas relacionados al transporte como: el exceso de tráfico, la negligencia de los chóferes, el mal servicio que se brinda y la falta de respeto al usuario de estas empresas de servicio público.

Desde hace tiempo se quiso encontrar la solución para mejorar el sistema vial limeño, es así que surge la opción de EL METROPOLITANO, según sus creadores, un sistema moderno, rápido, práctico que cambiaria nuestra cultura vial.

Es así que se inicia su construcción e implementación, esperándose instaurar un orden y mejorar el sistema de transporte público, pero surgieron posteriormente problemas que incomodaron a gran parte de la población aledaña a sus rutas; y es que según disposiciones municipales, muchas rutas que eran masivamente concurridas por los usuarios, fueron desviadas, imponiendo a la población una única opción: usar solo el Metropolitano. Sin duda, las personas que ya tenían una costumbre se vieron afectadas al ser obligadas a utilizarlo como su única ruta vial, surgiendo además quejas por la alta tarifa del servicio.

En el presente trabajo, se analizara las características del mercado que ha sido afectado por la implementación del metropolitano y se determinara si este sistema está en vías de convertirse en un monopolio.

\section{Palabras Clave:}

Sistema de transporte, transporte público, metropolitano, mercado, monopolio.

\begin{abstract}
Currently, in our capital, problems arise related to transportation, as excess traffic, the negligence of drivers, poor service provided and the lack of respect to the user of these public service companies.

It has long been wanted to find the solution to improve the road system Lima, then arise the option of THE METROPOLITAN, according to its creators, a modern system, faster, practical, that would change our vial culture. Thus begins its construction and implementation, and will be expected to establish an order and improve the public transport system, but arose problems that bothered to much of the population near of its routes, because of municipal regulations, since many routes that attended to several users, were diverted. It imposed to the population a single option: use only The Metropolitan. Certainly, people who already had a custom were affected by being forced to use it as their only road vial. In addition, complaints arose about the high cost of service.

In this paper, we analyze the characteristics of the market that has been affected by the implementation of the Metropolitan and determine whether the system is on track to become a monopoly.
\end{abstract}

Keywords: Transport system, public transport, Metropolitan, Market, Monopoly.

\footnotetext{
* Doctora en Ciencias Económicas. Ex- Vicerrectora Administrativa- UNMSM. Docente Investigadora de la UNMSM.
} E-mail: bherrerag@unmsm.edu.pe 


\section{METODOLOGÍA}

\section{Planteamiento del Problema}

¿El metropolitano está en vías de ser un monopolio?

Muchas opiniones hay en conformidad con la construcción del metropolitano, y es que no se puede negar que este sistema es ordenado y rápido, favoreciendo en muchos casos a estudiantes y trabajadores; pero desde su creación se viene creando una atmosfera de disconformidad y es que la eliminación de rutas que cumplían su normal función a causado el malestar de los transeúntes, sintiéndose obligados a utilizar este medio.

Ahora, nosotros no nos podemos basar en simples suposiciones de la población, es por ello que para determinar si es cierto que se está formando un monopolio, nos hemos visto en la necesidad de utilizar el índice de $\mathrm{HH}$, para así, con sus resultados poder analizar y determinar si es veraz afirmar que el metropolitano será, en tiempo más, un monopolio.

Por ello, hemos determinado que el presente trabajo reunirá, no solo investigación teórica, si no que tendrá opiniones de diferentes personas que se encuentran aledañas al metropolitano, para así determinar el tipo de mercado actual.

\section{OBJETIVOS}

- Objetivo General:

Comprobar si el sistema de transporte el Metropolitano está en vías de convertirse en un monopolio.

- Objetivos Específicos:

Determinar el tipo de mercado que se ha dado con implementación del sistema de transporte.

Conocer y determinar como la imple- mentación del sistema de transporte del Metropolitano, afectó a los consumidores.

Analizar el cambio de mercado y determinar sus consecuencias.

\section{HIPÓTESIS}

Consideramos que el sistema de transporte el Metropolitano está en vías de ser un monopolio debido a los últimos acontecimientos relacionados con este medio, como la desviación y /o eliminación de rutas de algunos medios de transporte afectando la decisión de los consumidores.

Para la elaboración de esta hipótesis nos basamos en las siguientes variables:

\section{Variable 1:}

Con la ampliación de rutas del metropolitano, muchas líneas de transporte han sido desviadas formándose así un mercado con pocas opciones para los consumidores.

\section{Variable 2:}

Las personas, debido a la desviación y/o eliminación de las líneas de transporte se han visto obligados a utilizar el metropolitano como medio de transporte urbano.

\section{MARCO TEÓRICO}

\section{MERCADO}

Desde el punto de vista de la economía, El mercado es cualquier conjunto de transacciones o acuerdos de negocios entre compradores y vendedores. En contraposición con una simple venta, el mercado implica el comercio formal y regulado, donde existe cierta competencia entre los participantes.

El mercado es, también, el ambiente social que propicia las condiciones para el intercambio. Se debe de interpretar como la institución u organización social a través de 
la cual los vendedores y compradores de un determinado bien o servicio, entran en estrecha relación comercial a fin de realizar abundantes transacciones comerciales.

\section{Tipos de mercado}

Conocer el tipo de mercado es muy útil porque permite identificar el contexto de mercado en función a su ubicación geográfica, los tipos de clientes que existen, la competencia establecida, el tipo de producto, el tipo de recursos y los grupos de clientes.

Los mercados en donde operan las empresas varían mucho entre sí. Algunos son altamente competitivos, lo que provoca que obtener beneficios sea muy difícil.

Otros están aparentemente libres de competencia y las empresas que participan en ellos obtienen grandes beneficios.

Otros más están dominados por feroces campañas publicitarias a través de la que cada empresa trata de persuadir a los consumidores que ellos tienen los mejores productos.

Los tipos de mercado se clasifican de acuerdo al número de ofertantes y demandantes:

\section{Mercado de competencia imperfecta}

Una situación del mercado en la que los vendedores o empresas que compiten en él, tienen cierto control sobre el precio debido a que ofertan productos diferenciados $\mathrm{y} / \mathrm{o}$ limitan el suministro. Además, en este tipo de mercado existe información incompleta del mercado y comportamiento emocional de compra, por lo que las empresas utilizan la promoción para informar, persuadir o recordar a su mercado meta las características y beneficios de sus productos. El monopolio, el oligopolio y la competencia monopolística, son los tres tipos de competencia imperfecta que existen en la actualidad.

\section{Tipos de competencia imperfecta:}

Competencia monopolística

Ocurre cuando un gran número de vendedores produce bienes diferenciados a precios algo distintos y en la que ninguno posee una gran cuota de mercado.

La tendencia de las empresas en los sistemas de economía de mercado es la de buscar la ventaja competitiva, es decir, una posición dominante en el mercado en el que actúan. Esto ha llevado a algunas de ellas a diferenciar los productos o servicios que ofrecen tratando de vender un bien o un servicio en régimen de monopolio o cuasi monopolio.

\section{Monopolio}

Es una industria que produce un bien o servicio para el que no existen sustitutos cercanos $y$ en donde hay un solo vendedor, protegido de la competencia por una barrera que evita la entrada de nuevas empresas a la industria pues es ello la causa fundamental para que exista el monopolio.

\section{Características del monopolio:}

- No hay sustitutos cercanos: aun cuando una sola empresa produzca un bien, si dicho bien tiene un sustituto cercano, esa empresa enfrenta la competencia de los productores de los sustitutos.

- Los monopolios están constantemente, bajo el ataque de nuevos productos e ideas que pueden sustituir sus productos

- Barreras a la entrada de nuevas empresas: son las restricciones legales o naturales que protegen a una empresa de la posible competencia

- Barreras legales a la entrada: crea un monopolio legal, es decir, es un mercado en donde la competencia y la entrada de nuevas empresas están restringidas por la concesión de una franquicia pública, una 
licencia gubernamental, una patente o por derechos de autor.

- Franquicia pública: es un derecho exclusivo que se concede a una empresa para ofrecer un bien o servicio.

- Licencia gubernamental: controla la entrada a ocupaciones, profesiones e industrias particulares. Las licencias no siempre crean monopolio, pero si restringen la competencia

- Barreras naturales a la entrada: crea un monopolio natural, es decir una industria en donde una empresa puede proveer un bien o un servicio al mercado completo a un precio menor del que ofrecía 2 o más empresas.

\section{Tipos de Monopolio}

\section{Monopolio natural o puro}

El monopolio puro es cuando existe una única empresa en una industria; no suele darse en la economía real, excepto cuando se trata de una actividad desempeñada mediante una concesión pública.

Existe un monopolio puro solo si hay un único vendedor en un mercado bien definido con muchos compradores. En éste caso no existe rivalidad personal, ni competitividad empresarial, por la sencilla razón que no hay rivales.

Sin embargo, las políticas de un monopolista podrían verse restringidas por la competencia indirecta de todos los bienes por el dinero del consumidor, así como por la competencia de los bienes que sean sustitutos razonablemente adecuados y por la amenaza de una competencia potencial si es posible la entrada en el mercado.

Un monopolista puro es la única empresa en la industria y se enfrenta a la curva de demanda de la industria, la cual, necesariamente, presenta pendiente negativa.

\section{Monopolio artificial}

Un monopolio artificial es un tipo de monopolio en el cual el monopolista se vale de algún medio para impedir que vayan al mercado más productos que los suyos. Los medios de los que se puede valer pueden ir desde la violencia a una fuerte restricción de la demanda por parte de los consumidores, pasando por la imposición de barreras de entrada artificiales o de regulación (ver Monopolio de la violencia). Estos últimos, tienen un carácter fiscal. El Estado puede reservarse de forma exclusiva algunas producciones $u$ otorgar privilegios a ciertas empresas o mantener un régimen de concesión de licencias restrictivo o proteger patentes y derechos de autor esenciales para realizar la actividad.

\section{Monopolio natural}

Un monopolio natural es un caso particular en el cual una empresa que puede producir toda la producción que necesita el mercado con un coste menor que si hubiera varias empresas compitiendo.

Esto usualmente ocurre en mercados donde las firmas tienen que realizar una altísima inversión inicial para ingresar (economías de escala). Los incentivos para que otras firmas ingresen son nulos y, por otro lado, también sería ineficiente, es decir, resulta más eficiente que sólo haya una empresa en el sector puesto que los costes medios tienden a 0 según se aumenta la cantidad haciendo el negocio más rentable y ayudando la monopolista a bajar el precio.

\section{Monopolio discriminador de precios}

El monopolio discriminador de precios es aquél que pone diferentes precios según las características del mercado, del producto o de los consumidores para obtener mayor beneficio económico.

La forma ideal de la discriminación de precios es cobrar de cada comprador lo máxi- 
mo que el comprador está dispuesto a pagar. Así la curva de ingreso marginal se convierte en idéntica a la curva de la demanda. El vendedor va a vender la cantidad económicamente eficiente, capturando la totalidad del excedente del consumidor y aumentando sustancialmente los beneficios.

\section{Vías para la aparición de un monopolio.}

Estas fuentes de poder de mercado pueden terminar formando una estructura monopolística. De entre todas las vías que pueden desencadenar la aparición de un monopolio se puede destacar:

\section{Trust}

Este tipo de convenios permiten transferir el control real de una empresa a un individuo o a otra empresa, intercambiando las acciones por certificados emitidos por los individuos que pretenden controlar la empresa.

Estos acuerdos no tienen por qué ser ilegales, excepto cuando se adoptan con el fin de monopolizar el comercio.

\section{Carteles}

Es un acuerdo formal entre empresas del mismo sector, cuyo fin es reducir o eliminar la competencia en un determinado mercado. Los cárteles suelen estar encaminados a desarrollar un control sobre la producción y la distribución de tal manera que mediante la colusión de las empresas que lo forman éstas forman una estructura de mercado monopolística, obteniendo un poder sobre el mercado en el cual obtienen los mayores beneficios posibles en perjuicio de los consumidores. Sus principales actividades se centran en fijar los precios, limitar la oferta disponible, dividir el mercado y compartir los beneficios.

\section{Fusiones}

Las fusiones son intentos de organizar la industria producidas por la búsqueda de economías de escala o de ventajas monopóli- cas; en el primer caso son una expresión del proceso de integración horizontal o vertical entre empresas independientes, que buscan una mayor eficiencia en sus procesos productivos; en el segundo caso pueden considerarse como una forma de control de los mercados más profunda y orgánica que la formación de cárteles.

\section{MEDIDAS DE CONCENTRACIÓN DE MERCADO}

Las medidas de concentración tratan de resaltar el mayor ó menor grado de equidad en la distribución total de algún bien, servicio o ingreso. Son, por tanto, indicadores del grado de distribución de éstos.

Para darnos una idea aproximada del grado de competencia existente en un mercado se definen las medidas de concentración. Los usados más frecuentemente son el índice de concentración y el índice de Herfindahl o Herfindahl-Hirshman).

Para medir la concentración se han desarrollado una gran cantidad de índices, conocidos coloquialmente como Índices de Concentración, entre los índices más conocidos tenemos; Índice de Entropía, Índice Exponencial, Índice de Herfindal, Índice de Herfindal, Índice de Gini y Curva de Lorenz.

\section{El coeficiente de concentración de las 4} empresas

También llamada CC4E, es el porcentaje del valor de las ventas de una industria que corresponde a las cuatro empresas más grandes de ese ramo. El rango del coeficiente de concentración va de casi cero para la competencia perfecta hasta el 100 por ciento para el monopolio. Este coeficiente es una de las medidas más utilizadas para evaluar la estructura de un mercado.

Un coeficiente de concentración bajo señala un alto grado de competencia y un coefi- 
ciente de concentración alto señala ausencia de competencia. Sus parámetros son:

${ }^{*}$ Un monopolio: $100 \%$ de concentración de mercado.

*Un CC4E mayor de $60 \%$, indica un mercado altamente concentrado y se define como oligopolio.

*Un CC4E menor de 40\%, indica un mercado competitivo.

\section{El índice de Herfindahl- Hirschman}

También llamado el IHH es una medida de uso general en economía de la concentración económica en un mercado. $\mathrm{O}$, inversamente, la medida de falta de competencia en un sistema económico. A más alto el índice, más concentrado, menos competitivo el mercado. El índice se calcula elevando al cuadrado la cuota de mercado que cada empresa posee y sumando esas cantidades. Los resultados van desde cerca a 0 (competencia perfecta) a 10.000 (control monopólico).

Por ejemplo, si un monopolio que controle la totalidad (100\%) del mercado, 100 elevado al cuadrado es 10.000, dando un índice de 10 mil. Dos empresas que compartan igualmente el mercado: 50\% del mercado cada una es 2.500 cada una. Sumando esas cantidades nos da un índice de 5 mil. Cuatro empresas con control del mercado de $30 \%, 30 \%, 20 \%$ y $20 \%$ respectivamente nos da $302+302+202$ $+202=2600$

Lo anterior se puede resumir y expresar matemáticamente de la siguiente manera:

Es decir: el índice es la suma de las "cuotas de mercado" al cuadrado de las n empresas que lo componen.

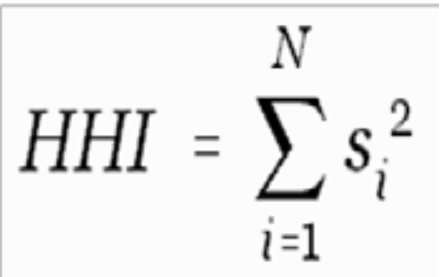

\section{MARCO HISTÓRICO}

\section{ANTECEDENTES DEL MERCADO ANTES DE LA CONSTRUCCIÓN DEL METROPOLITANO}

Su construcción se inició en el año 2006, durante la gestión edil del ex alcalde Luis Castañeda Lossio y su operación comercial inició el 28 de julio del 2010 de manera parcial. El corredor cubre una ruta segregada Corredor Segregado de Alta Capacidad (COSAC) que de sur a norte comprende 16 distritos limeños desde Chorrillos hasta Independencia. La longitud de esta ruta troncal es de $26 \mathrm{~km}$ y el número total de estaciones es de 38 ; además se complementa con rutas alimentadoras en sus extremos sur $y$ norte.

\section{GESTACIÓN DEL METROPOLITANO}

\section{ETAPAS DE DESARROLLO:}

\section{a) COSAC I:}

En abril de 2007 se inició la construcción de éste proyecto, con el cierre del carril central de la Vía Expresa de la Av. Paseo de República, para aumentar el número de carriles, construir los paraderos del futuro corredor vial y desmontar los puentes innecesarios. Así mismo, también se comenzó la construcción de las ciclo vías en el tramo Corredor Sur. En julio comienza la construcción de las vías troncales en el mismo tramo. El 16 de julio comenzó la construcción de la Estación Central Grau.

En mayo de 2008, se inició la excavación de los túneles subterráneos que conectan a la Estación Central con las Vías Expresas de Paseo de la República y Avenida Grau.[1].

El 1 de mayo de 2010 se inicia una operación blanca desde la estación Matellini hasta la Estación Central, probando la operación 
con 20 buses articulados, de los cuales solo algunos recogían pasajeros, sin cobrar aun la tarifa.

El 28 de julio de 2010 se inicia la operación formal pagada del servicio en la troncal sur (de la Estación Central a la Terminal Matellini). Las rutas alimentadoras del sur y los usuarios de tarjetas preferenciales (escolares, universitarios y de institutos reconocidos por el Ministerio de Educación que recorran la troncal centro - sur y los alimentadores) seguirán siendo gratuitas hasta mediados de Agosto.

El 2 de agosto de 2010 empiezan las pruebas en vacío de los dos corredores centro: A (Lampa - Emancipación - Plaza Castilla) y B (España - Alfonso Ugarte - Dos de Mayo), hasta Caquetá.

El 11 de agosto inició la prueba gratuita con pasajeros en la ruta Centro B: Estación Central - España - Alfonso Ugarte - 2 de Mayo, hasta Caquetá y viceversa, dando la vuelta en el Parque del Trabajo.

El 24 de agosto inicia la marcha blanca con pasajeros en la ruta Centro A: Estación Central - Lampa - Emancipación - Plaza Ramón Castilla, hasta Caquetá y viceversa, dando la vuelta en el Parque del Trabajo.

El 5 de septiembre se dio inicio a las pruebas en vacío del corredor norte, desde la Estación Central a la Terminal norte Naranjal. Los buses que llegaban con pasajeros a la Estación Caquetá por cualquiera de las dos rutas del corredor centro, eran desembarcados en dicha estación para proseguir a la Terminal norte Naranjal en vacío y retornar.

El 11 de septiembre empezó la prueba con pasajeros del corredor norte, desde la Estación Central a la Terminal Naranjal $y$ viceversa, a través del corredor centro $B$. También por el corredor centro A, haciendo transbordo en la estación Caquetá para proseguir. Poco después, los buses de la ruta $\mathrm{A}$ llegan a la terminal Naranjal sin necesidad de transbordo en Caquetá.

El 2 de octubre inició la operación formal pagada de los tramos centro y norte, completando toda la ruta la troncal de este servicio.

El 16 de diciembre inició la operación comercial de los alimentadores del norte en la terminal Naranjal, luego de casi un mes de pruebas sin pasajeros. Ello, sumado a la puesta en marcha de la estación "Jirón de la Unión” en la ruta centro A (la última estación pendiente de la troncal) y de la próxima circulación del total de la flota de buses articulados, el Metropolitano opera al 100\% de su capacidad.

En declaraciones al diario La República el 18 de Julio, Juan Tapia Grillo, presidente del directorio de Protransporte (empresa municipal que administra el Metropolitano), informó que se han iniciado los estudios definitivos de ingeniería para que la línea 1 crezca 7.5 kilómetros más, desde la estación Naranjal, en Independencia, hasta el extenso patio "Sinchi Roca", ubicado en Comas, según el plan original. Anunció que no solo continuará su trayecto por la avenida Túpac Amaru, sino que llegará hasta el kilómetro 24.5 de la Panamericana Norte, hasta el cruce con la avenida Canta Callao, en San Martín de Porres. Estaría listo a fines del 2012 con una inversión de 100 millones de dólares. 1

\section{b) COSAC II:}

En conferencia de prensa del 2 de diciembre de 2010, Susana Villarán de la Puente, alcaldesa electa de Lima, anunció que la línea 2 del Metropolitano se hará al inicio de su gestión. Esta nueva ruta unirá Ate (Av. Grau, av. Nicolás Ayllón y av. Carretera Central) con el Callao (Av. España, última cuadra de la av. Alfonso Ugarte, Plaza Francisco Bolognesi, av. Arica y Av. Venezuela), pasando por la Estación Central. Interconectará con la línea 1 
del Metro de Lima, en la estación intermodal Grau.

Juan Tapia Grillo anunció que a mediados del 2012 se hará la licitación de la obra, para el inicio de las mismas a fines del mismo año. Estaría concluido y en servicio en el 2014.

\section{2. ¿Qué es el Metropolitano?}

Con buses articulados de gran capacidad que circulan por corredores exclusivos, bajo el esquema de autobuses de tránsito rápido $\mathrm{BRT}$ (Bus Rapid Transit en inglés).

El sistema de autobús de tránsito rápido (Bus Rapid Transit en inglés, BRT) es un término dado a una amplia variedad de soluciones de transporte basada en autobuses.

El propósito es el de proporcionar infraestructura vial que permita alcanzar una alta capacidad de usuarios y un servicio de bus de alta calidad con respecto al servicio tradicional de bus urbano.

Se trata de un sistema de buses de alto estándar que combina el diseño de estaciones, vehículos, tecnología y planificación orientada a los usuarios

El propósito es el de proporcionar infraestructura vial que permita alcanzar una alta capacidad de usuarios y un servicio de bus de alta calidad con respecto al servicio tradicional de bus urbano.

Se trata de un sistema de buses de alto estándar que combina el diseño de estaciones, vehículos, tecnología y planificación orientada a los usuarios.

\section{Objetivo del METROPOLITANO:}

El objetivo de este moderno sistema es elevar la calidad de vida de los ciudadanos, al ahorrarles tiempo en el traslado diario, proteger el medio ambiente, brindarles mayor segu- ridad, una mejor calidad de servicio y trato más humano, especialmente a las personas de la tercera edad y con discapacidad.

El Metropolitano es un sistema creado para favorecer al empresario nacional de transporte formal, dándole la posibilidad de desarrollar y operar su empresa y obtener las ganancias que son fruto de su esfuerzo. De igual manera, el Metropolitano también va a darle mayor movilidad, seguridad y confiabilidad a los sectores menos favorecidos de la población urbana para que ellos puedan acceder a mayores oportunidades de empleo y servicios en la ciudad.

\section{CARACTERÍSTICAS DEL SERVICIO DEL METROPOLITANO}

\section{Ruta:}

La ruta que sigue el corredor Vial empieza en el distrito de Independencia, al Norte de la ciudad, y va parte de la Avenida Túpac Amaru hasta la Avenida Caquetá, de ahí sigue hasta la Plaza Castilla donde se bifurca: por un lado sigue hacia la Plaza Dos de Mayo hasta la Avenida España e ingresa a la Estación Central Grau; de otro lado va hacia el Jirón de Lampa a través de la Avenida Tacna, una vez allí toma dirección hacia la Estación Central Grau.

Desde la Estación Central Grau toma dirección hacia el Sur a través de lo que hoy en día es la Vía Expresa de Paseo de la República hasta su final, luego sigue con dirección hacia el Ovalo José Balta, sigue hacia la Municipalidad de Barranco, el Estadio Municipal de ese distrito, para después llegar a la Escuela Militar de Chorrillos, una vez allí sigue para finalizar su recorrido en la Urbanización Matellini, cerca al límite del distrito de Chorrillos con Surco. 


\begin{tabular}{|c|c|}
\hline Estación Caquetá: & Av. Caquetá codra. 1 con Av. Zarumilla cdra. 1, San Martín de Porres - Rimac \\
\hline Estación Parque de & Av. Caquetá codra. 7 con Antón Sảnchez codra. 1, San Martín de Porres - Rimac \\
\hline Estación Uni: & Av. Túpac Amaru cdra. 2, San Martin de Porres - Rimac \\
\hline $\begin{array}{l}\text { Estación Honorio } \\
\text { Delgado: }\end{array}$ & $\begin{array}{l}\text { Av. Túpac Amaru cdra. } 8 \text { con Av. Honorio Delgado cdra. 1, San Martín de Porres - } \\
\text { Independencia }\end{array}$ \\
\hline Estación El Milagro & $\begin{array}{l}\text { Av. Túpac Amaru cdra. } 14 \text { con Av. Bartolomé de las Casas cora. 1, San Martín de } \\
\text { Porres - Independencia }\end{array}$ \\
\hline $\begin{array}{l}\text { Estación Tomas } \\
\text { Valle: }\end{array}$ & $\begin{array}{l}\text { Av. Túpac Amaru (Av. Las Violetas cdra. 1) con Av. Tomas Valle cdra. } 1, \\
\text { Independencia }\end{array}$ \\
\hline Estación Jazmines: & Av. Túpac Amaru cdra. 72 con Av. Los Jazmines cdra. 1, Independencia \\
\hline Estación & Av. Túpac Amaru cdra. 33 con Av. Los Pinos cdra. 1, Independencia \\
\hline Estación Pacifico: & Av. Túpac Amaru cdra. 35 con Av. Pacifico cdra. 1, Independencia \\
\hline Estación Izaguirre: & Av. Túpac Amaru cdra. 37 con Av. C. Izaguirre cdra. 1, Independencia \\
\hline Estación Naranjal: & Av. Túpac Amaru cdra. 45 con Av. Chinchaysuyo cdra. 1, Independencia \\
\hline
\end{tabular}

\section{ESTACIONES}

\section{TRONCAL NORTE}

Al cruzar el Puente del Ejército el bus ingresa por la Av. Caquetá entre los distritos de San Martín de Porres y el Rímac, luego dobla a la izquierda en el inicio de la Av. Túpac Amaru y la recorre en su totalidad, entrando al distri- to de Independencia, hasta el Óvalo Naranjal donde se encuentra la Estación de Transferencia Naranja

La ruta alimentadora norte es aquella que te lleva:

$\checkmark \quad$ De tu paradero inicial hacia la Estación de Transferencia Naranjal.

$\checkmark$ De la Estación de Transferencia Naranjal hacia tu paradero final.

\begin{tabular}{|c|c|}
\hline Estación Central: & $\begin{array}{l}\text { Parque Los Héroes Navales con Av. Paseo de la República 170, Cercado } \\
\text { de Lima }\end{array}$ \\
\hline Estación Colmena: & $\begin{array}{l}\text { Av. Nicolás Piérola (Colmena) cdra. } 11 \text { con Jr. Lampa cdra. 9, Cercado de } \\
\text { Lima }\end{array}$ \\
\hline Estación Jirón de la Unión: & Av. Emancipación cdra. 1 con Jr. De la Unión cdra. 6, Cercado de Lima \\
\hline Estación Tacna: & Av. Emancipación cdra. 4 y 5 con Av. Tacna cdra. 5, Cercado de Lima \\
\hline Estación España: & $\begin{array}{l}\text { Av. Alfonso de Ugarte cdra. } 13 \text { con Av. Republica de Portugal, Breña - } \\
\text { Cercado de Lima }\end{array}$ \\
\hline Estación Quilca: & $\begin{array}{l}\text { Av. Alfonso de Ugarte cdra. } 9 \text { con Jr. Pomabamba, Breña - Cercado de } \\
\text { Lima }\end{array}$ \\
\hline Estación 2 de Mayo: & Av. Alfonso Ugarte cdra. 4 y 5 con Plaza Dos de Mayo, Cercado de Lima \\
\hline Estación Ramón Castilla: & Av. Emancipación cdra. 9 con Jr. Tayacaja cdra. 6, Cercado de Lima \\
\hline
\end{tabular}


Se cuenta con once rutas alimentadoras norte:

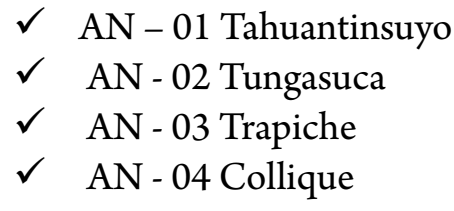

\begin{tabular}{|c|c|}
\hline Estación Matellini: & $\begin{array}{l}\text { Av. Prolongación Paseo de la República cdra. } 18 \text { con Av. Ariosto Matellini, } \\
\text { Chorrillos }\end{array}$ \\
\hline Estación Rosario de Villa: & Av. Prolongación Paseo de la República cdra. 8 con Jr. Costa Azul, Chorrillos \\
\hline Estación Terán: & $\begin{array}{l}\text { Av. Prolongación Pas eo de la República cdra. } 1 \text { conAv. Fernando Terán cdra. } \\
\text { 9. Chorrillos }\end{array}$ \\
\hline Estación Escuela Militar: & Av, Escuela Militar cora. 8, Chorrillos \\
\hline Estación Estadio Unión: & Av. Escuela Militar cdra. 2 con Pasaje Anaya, Barranco \\
\hline Estación Bulevar: & Av. R. Panamá (Bolognesi) cdra. 3 con Calle Juan Pazos, Barranco \\
\hline Estación Balta: & Av. R. Panamá (Bolognesi) cdra. 10 con Ovalo Balta, Barranco \\
\hline Estación Plaza de Flores: & Av. Rosendo Vidaurre entre las cuadras 5 y 6 , Barranco \\
\hline Estación 28 de Julio: & Av. Paseo de la República cdra. 61 con Puente Miraflores, Miraflores \\
\hline Estación Benavides: & Av. Paseo de la República cdra. 59 con Puente Benavides, Miraflores \\
\hline Estación Ricardo Palma: & $\begin{array}{l}\text { Av. Paseo de la República cdra. } 55 \text { con Puente Ricardo Paima, Miraflores - } \\
\text { Surquillo }\end{array}$ \\
\hline Estaciôn Angamos: & Av. Paseo de la República cdra. 48 con Puente Angamos, Miraflores - Surquillo \\
\hline Estación Domingo Orué: & $\begin{array}{l}\text { Av. Paseo de la República codra. } 43 \text { con Puente Domingo Orué, Miraflores - } \\
\text { Surquillo }\end{array}$ \\
\hline Estación Aramburú: & Av. Paseo de la República cdra. 37 con Puente Aramburú, San Isidro \\
\hline $\begin{array}{l}\text { Estación Canaval } \\
\text { Moreyra: }\end{array}$ & yAv, Paseo de la República cdra. 32 con Puente Canaval y Moreyra, San Isidro \\
\hline Estación Javier Prado: & Av. Paseo de la República cdra. 26 con Puente Javier Prado, San Isidro \\
\hline Estación Canadá: & Av. Paseo de la República cdra. 19 con Puente Canadá, Lince - La Victoria \\
\hline Estación México: & $\begin{array}{l}\text { Av. Paseo de la República cura. } 13 \text { con Puente México, Cercado de Lima - La } \\
\text { Victoria }\end{array}$ \\
\hline Estación Estadio Nacio & $\begin{array}{l}\text { I: Av. Paseo de la República codra. } 9 \text { con Puente Peatonal, Cercado de Lima - La } \\
\text { Vietoria. }\end{array}$ \\
\hline Estación Central: & $\begin{array}{l}\text { Parque Los Héroes Navales con Av. Paseo de la República 170, Cercado de } \\
\text { Lima }\end{array}$ \\
\hline
\end{tabular}

$\begin{array}{ll}\checkmark & \text { AN - 05 Payet } \\ \checkmark & \text { AN - 06 Puno } \\ \checkmark & \text { AN - 08 Milagro de Jesús } \\ \checkmark & \text { AN - 10 Santo Domingo } \\ \checkmark & \text { AN - 12 Puente Piedra }\end{array}$

$\checkmark$ AN - 05 Payet

$\checkmark$ AN - 08 Milagro de Jesús

$\checkmark$ AN - 12 Puente Piedra
AN - 13 La Ensenada

AN - 14 Bertello

$\checkmark$ AN - 15 Los Alisos

$\checkmark$ AN - 16 Los Olivos

AN - 17 Antúnez de Manolo 


\section{ESTACION CENTRAL}

Desde la Estación Central subterránea, las vías segregadas se bifurcan una por el Jirón Lampa y otra por la Av. España:

$\checkmark \quad$ Las vías del Jirón Lampa vuelven a la superficie pasando la Av. Roosevelt, donde luego giran a la izquierda en la Av. Emancipación y la recorren hasta la Plaza Castilla.

$\checkmark \quad$ Las vías de la Av. España vuelven a la superficie pasando el Jirón Washington y giran a la derecha en la Av. Alfonso Ugarte en dirección al norte.
Ambos tramos se unen en la Plaza Castilla y prosiguen su camino hacia el Puente del Ejército.

\section{TRONCAL SUR:}

Desde la Estación de Transferencia Matellini en Chorrillos, los buses recorren la Prolongación del Paseo de la República subiendo por la Av. Escuela Militar, entrando a Barranco por la Av. Bolognesi, luego entrando a la Vía Expresa del Paseo de la República hasta llegar a la Plaza Grau en el Cercado de Lima donde se encuentra la Estación Central subterránea.

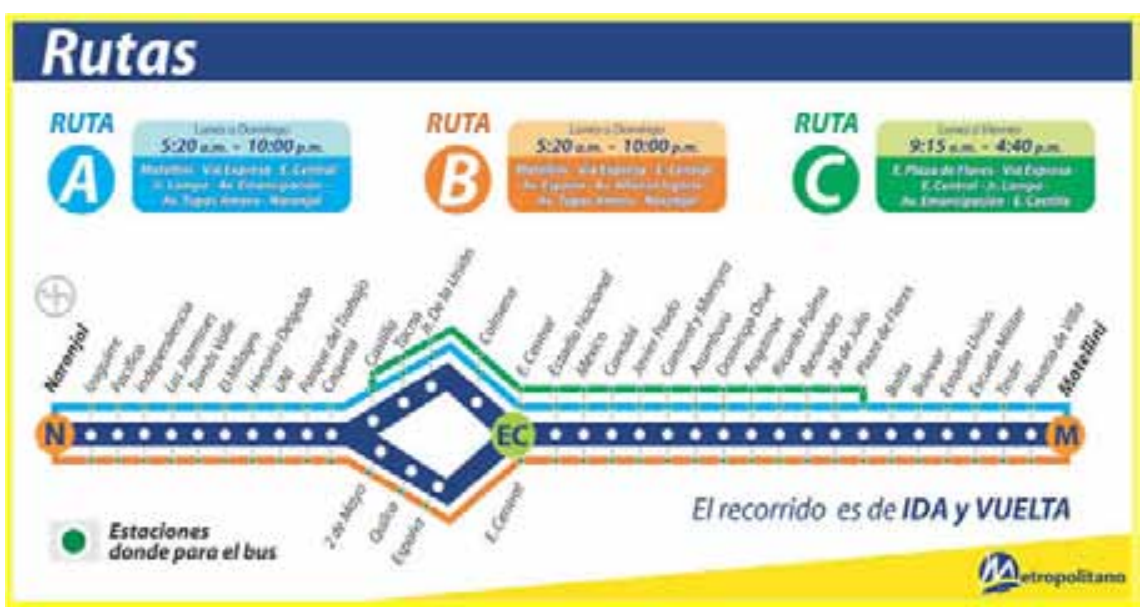

La ruta alimentadora sur es aquella que te lleva:

De tu paradero inicial hacia la Estación de Transferencia Matellini.

De la Estación de Transferencia Matellini hacia tu paradero final.

Se cuenta con cuatro rutas alimentadoras sur:

-AS - 02 Alameda Sur

-AS - 07 América

- AS - 08 Los Próceres

-AS - 09 Sagitario

\section{SERVICIOS:}

Actualmente hay dos tipos de servicios: El Regular y el Expreso.

\section{REGULARES:}

Se detienen en todas las estaciones. Se incluyen dentro de estos servicios el Regular A, el Regular B y el Regular C, determinados por su recorrido en la Troncal Centro. 


\section{$\checkmark$ Regular A:}

Circula por todas la estaciones de la Troncal Norte y la Troncal Sur, pero en la Troncal Centro solo pasa por la Ruta A. Es representado por el color celeste. El servicio es disponible de lunes a domingo de 06:00 a 22:00.

\section{$\checkmark$ Regular B :}

Circula por todas las estaciones de la Troncal Norte y la Troncal Sur, pero en la Troncal Centro solo pasa por la Ruta B. Es representado por el color anaranjado. El servicio es disponible de lunes a domingo de 06:00 a 22:00.

\section{$\checkmark$ Regular C:}

Circula por todas la estaciones desde la estación Plaza de Flores hasta la estación Ramón Castilla. En la Troncal Centro solo pasa por la Ruta A. Es representado por el color verde. El servicio es disponible de lunes a viernes de 09:15 a 17:00.

\section{EXPRESOS:}

Se detienen en estaciones preestablecidas, con la finalidad de lograr una mayor fluidez durante las horas puntas. Hay cuatro tipos de servicios Expresos. En la Troncal Centro los expresos solo pasan por la Ruta B.

\section{$\checkmark$ Expreso 1:}

El primer servicio (Expreso 1), se detiene en unas estaciones en ambas direcciones, viniendo desde el Terminal Sur Matellini. Es representado por el color plateado.

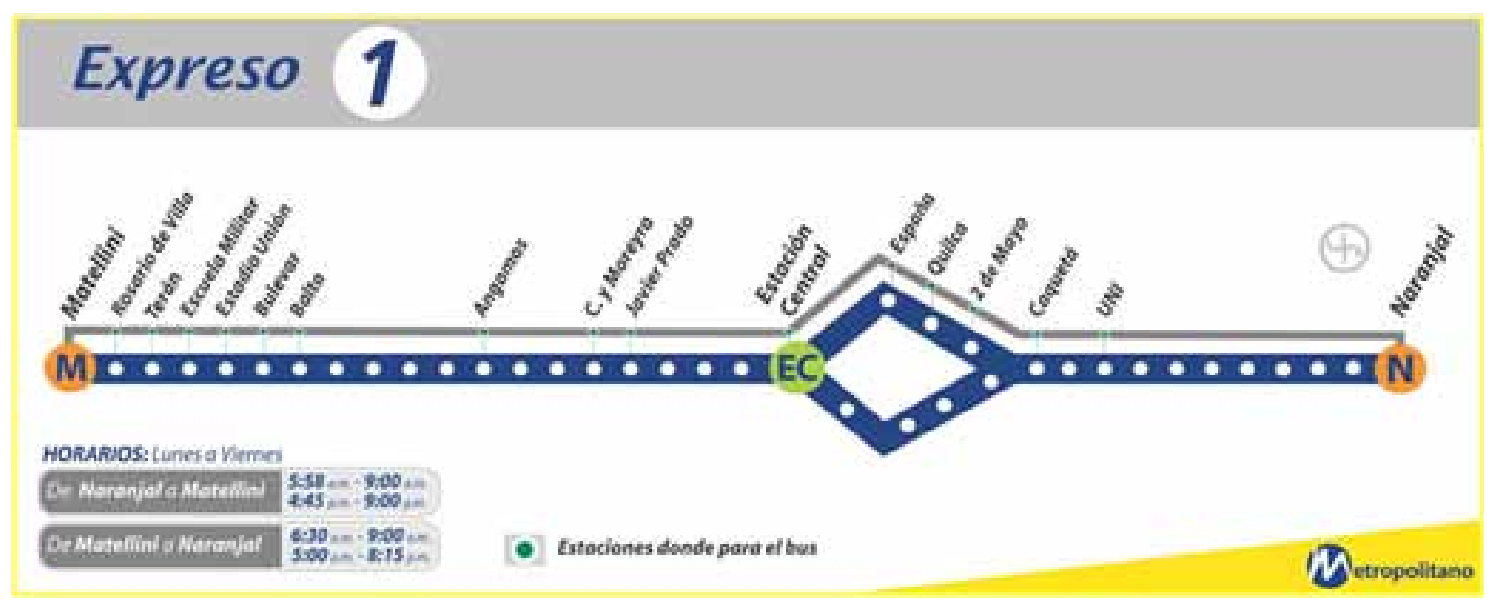

\section{$\checkmark$ Expreso 2:}

El segundo servicio (Expreso 2), estrenado el 8 de noviembre, no entra en los distritos de Barranco y Chorrillos donde existen limitaciones de sección vial en cuanto a adelanto de buses. Este se detiene en unas estaciones en ambas direcciones, partiendo desde el Terminal Norte Naranjal. Es representado por el color azul. 


\section{Expreso 2 Naranjal - Plaza de Flores - Naranjal}

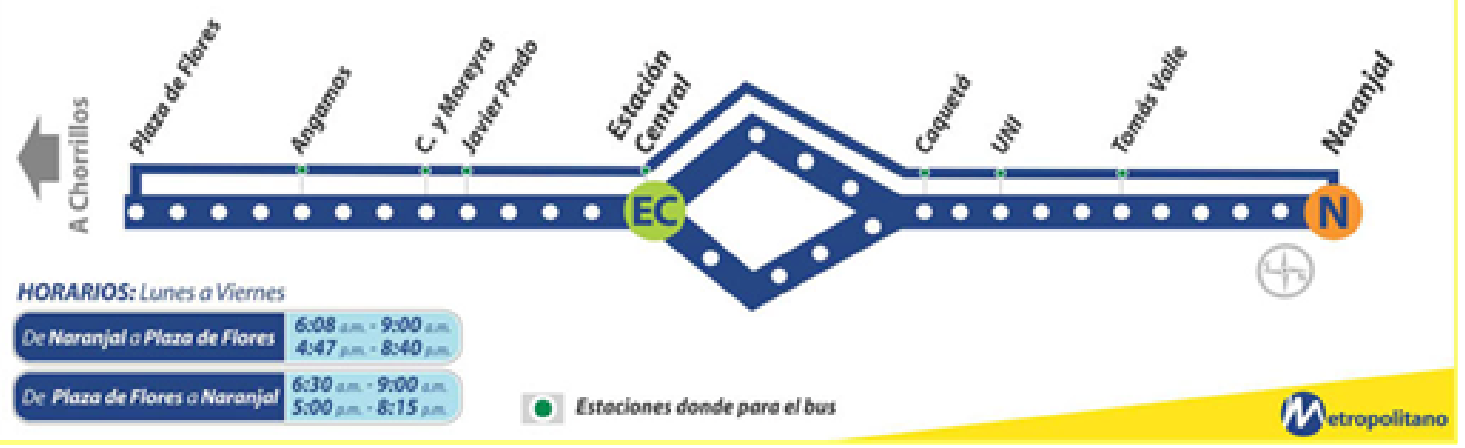

$\checkmark$ Expreso 3:

El tercer servicio expreso (Expreso 3), brinda un servicio exclusivamente para los usuarios de los distritos del norte de la ciudad que necesiten transportarse a Barranco y viceversa. Es representado por el color dorado.

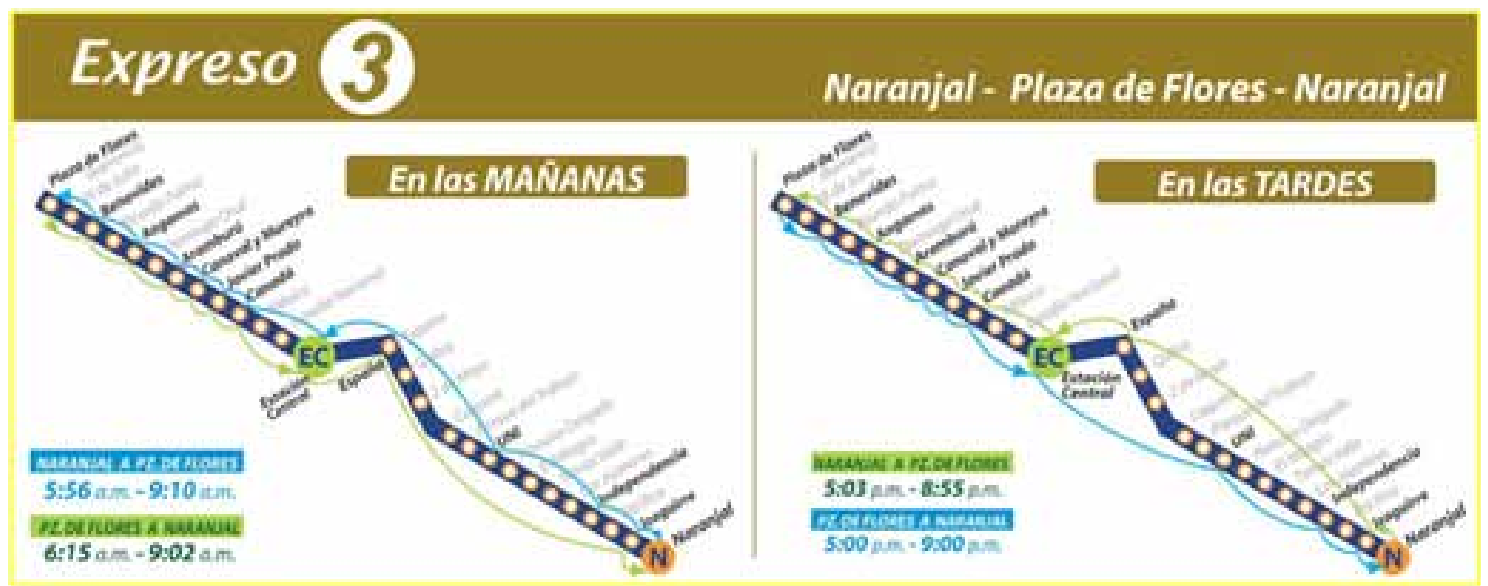

\section{$\checkmark$ Expreso 4:}

El cuarto servicio expreso (Expreso 4), brinda un servicio exclusivamente para los usuarios de los distritos del norte de la ciudad que necesiten transportarse a Barranco y viceversa. Funciona los sábados de 6:00 a 22:00 de forma ininterrumpida. Se detiene en unas estaciones en ambos sentidos, partiendo desde el Terminal Norte Naranjal. Es representado por el color rojo.

\section{Sistema Alimentador:}

Sistema Alimentador cubre exclusivamente el recorrido hacia las Estaciones de Transferencia Matellini y Naranjal y tiene como objetivo permitir el acceso de los usuarios a las Estaciones Intermedias o viceversa. Este servicio se toma en paraderos identificados.

\section{TARIFAS:}

La tarifa del Metropolitano es baja, debido a la competencia con otros servicios de transporte. El precio no sube por distancia, lo que significa que se pude bajar en cualquier estación. Los transbordos no tienen recargo alguno. 
Ruta troncal, precio

$$
\begin{array}{lll}
\checkmark & \text { Adulto } & \text { S/. } 1.50 \\
\checkmark & \text { Escolar } & \text { S/.0.80* } \\
\checkmark & \text { Universitario } & \text { S/.0.80 ** }
\end{array}
$$

Sistema alimentador, precio

$$
\begin{array}{lll}
\checkmark & \text { Adulto } & \text { S/.0.80 } \\
\checkmark & \text { Escolar } & \text { S/.0.40* } \\
\checkmark & \text { Universitario } & \text { S/.0.40 ** }
\end{array}
$$

* Válido solo en período escolar (Marzo a Diciembre), de lunes a viernes. Renovable, presentando documentación requerida.

** Válido de lunes a sábado, hasta la fecha de caducidad del carnet universitario o del instituto reconocido por el Ministerio de Educación. Renovable, presentando el carnet correspondiente.

\section{CONSECUENCIAS DEL METROPOLITANO}

\section{Consecuencias positivas}

- Después de la deviación de rutas por el norte la demanda de pasajeros aumento en 20 mil lo que generaría más ingresos y también conlleva a que aumenta la flota de carros en circulación.

- Se habilitar 30 kilómetros de ciclo vías en Chorrillos, Miraflores, San Isidro, Surco, Surquillo, Breña y Pueblo Libre, y que estas desemboquen en las estaciones del Metropolitano.

- Las personas llegan a su destino más rápido.

- Mas trabajo para los choferes ya que se requería de mucho personal para manejar los carros, los servicios de limpieza, etc.

\section{Consecuencias negativas}

Se desvió a 209 unidades de empresas como Chama, El Rápido y Arco Iris, ya que estos compartían un $30 \%$ del recorrido del Me- tropolitano. También en el sector norte del Metropolitano se desviaron 52 rutas (40 de Lima y 12 autorizadas por el Callao) a vías alternas para evitar que compitan con él.

Hubo mayor inversión de lo que se esperaba.

Otras consecuencias negativas de la construcción del Metropolitano es la destrucción de pistas internas a donde fue derivado el TRANSPORTE PUBLICO, CARGA PESADA; el caos vehicular y el riesgo de derrumbe de las viviendas que se ubican en las avenidas LIMA, GRAU, PIEROLA, BALTA Y SAN MARTIN

Las personas se sintieron obligadas a tomar el Metropolitano ya que desviaron los buses que tomaban antes.

\section{MERCADO ACTUAL DEL METROPOLITANO:}

SUR + CENTRO + NORTE (63)

Norte (20)

\section{Línea de tranporte que utiliza frecuentemente}

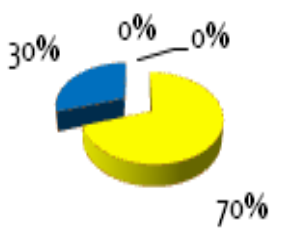

Metropolitano

notras líneas

Centro(29)

\section{Línea de transporte que utiliza...}

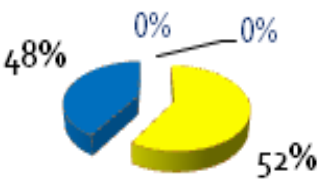

$\square$ Metropolitano

notras Líneas 


\section{Línea de transporte que utiliza...}

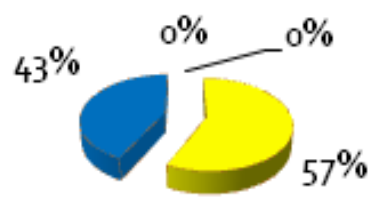

- Metropolitano

notras lineas

\section{RESULTADOS}

De las 63 personas encuestadas el 59\% si utilizando el metropolitano como medio de transporte, mientras que el resto del porcentaje prefiere otras líneas. De este 59\%, el 68\% se ve obligado a utilizar el Metropolitano debido a la desviación de sus rutas, teniendo como única opción este medio. El otro $32 \%$ no se ve obligado a utilizarlo debido a los precios módicos por las largas rutas que hacen y en otros casos por el buen servicio que reciben.

En el sector Norte, de los 20 encuestados el $70 \%$ si utiliza el metropolitano, mientras que un $30 \%$ no lo utiliza. De este $70 \%$, el $50 \%$ si se ve obligado a utilizar este medio, mientras que la otra mitad lo utiliza por decisión propia.

\section{¿Se vió forzado a utilizarlo?}

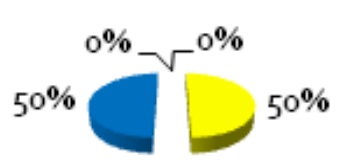

En el sector Centro, de los 29 encuestados el $52 \%$ utiliza el metropolitano, mientras que un $48 \%$ no lo utiliza. De este 52\%, el 33\% si se ve obligado a utilizar este medio, mientras que el $67 \%$ lo utiliza por decisión propia.

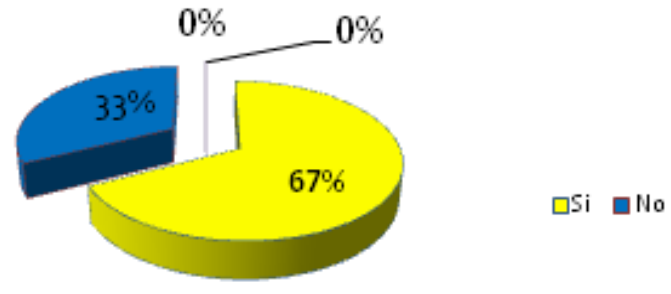

En el sector Sur, de los 14 encuestados el 57\% utiliza el metropolitano, mientras que un $43 \%$ no lo utiliza. De este $57 \%$, el $75 \%$ si se ve obligado a utilizar este medio, mientras que el $25 \%$ lo utiliza por decisión propia.

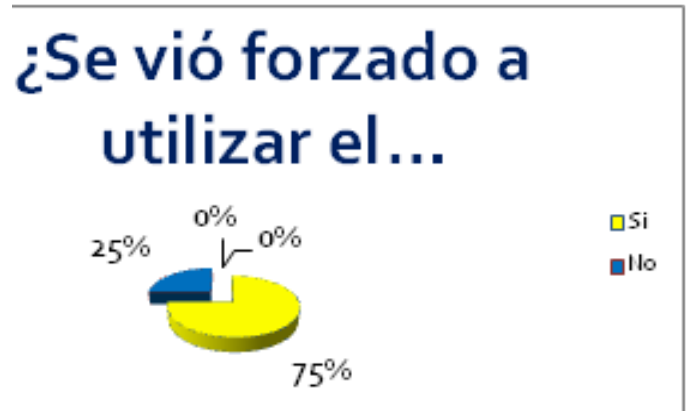

Mediante la medición de concentración del Índice de $\mathrm{HH}$, da como resultado, abarcando todos los sectores: 5162. Lo que evidencia un oligopolio en el mercado.

En el sector Norte, el Índice $\mathrm{HH}$ da como resultados: 5800, evidenciando un oligopolio.

En el sector Centro, el Índice $\mathrm{HH}$ da como resultados: 5008, evidenciando un oligopolio.

En el sector Sur, el Índice HH da como resultados: 5101.96, evidenciando un oligopolio.

Si bien es cierto que la brecha de porcentaje que separa al número de personas que utiliza el Metropolitano con las que no, no es muy grande; en el índice de $\mathrm{HH}$, el Metropolitano está teniendo la mayor concentración, mientras que el otro porcentaje está constituido por las diferentes líneas de transporte que las personas utilizan. 


\section{CONCLUSIONES}

1. Con las modificaciones para elevar el número de usuarios del metropolitano, se está aumentando las posibilidades para que este sea un monopolio.

1. En el aspecto positivo, los beneficiados con este cambio son los usuarios del metropolitano, ya que están ahorrando tiempo en recorrer largas distancias, $y$ en otros casos lo prefieren por el buen servicio que les brinda.

1. Por otro lado en el aspecto negativo, se está imponiendo a las personas el uso del metropolitano como única opción de medio de transporte, ya que las líneas que antes utilizaban han sido desviadas de sus habituales rutas.

1. Creo que antes de tomar cualquier decisión referente a la reforma sobre el transporte debe haber un consenso entre las partes para que así nadie se vea afectado y que los usuarios que cumplimos un papel crucial no nos veamos afectados.

\section{REFERENCIAS BIBLIOGRÁFICAS}

1. Microeconomía Versión para Latinoamérica 7ma Edición. Editorial Pearson. Autor: Michael Parkin, Gerardo Esquivel y Mercedes Muño.
2. http://www.protransporte.gob.pe/expresiones.html

3. http://www.protransporte.gob.pe/enlaces.html

4. http://www.metropolitano.com.pe/

5. http://www.uclm.es/area/fae/micro/ transparencias/5.pdf

6. http://www.wikipedia.com

7. http://www.gestiopolis.com

8. http://www.ecobachillerato.com/temaseco/temas/4clasesmercados.pdf

9. http://www.promonegocios.net/mercado/tipos-de-mercado.html

10. http://protransporte.gob.pe/index.html

11. http://docs.google.com/viewer? $\mathrm{a}=\mathrm{v} \&$ $\mathrm{q}=$ cache:rWnnis4hvcsJ:wwwoocities. org/espanol/panaley/organizacion produccion_mercados_eco100a_2008. $\mathrm{ppt}+\mathrm{el}+$ coeficiente $+\mathrm{de}+$ concentracio $\mathrm{n}+\mathrm{de}+\mathrm{las}+4+\mathrm{empresas} \& \mathrm{hl}=\mathrm{es} \& \mathrm{gl}=\mathrm{pe}$ \&pid=bl\&srcid=ADGEESjHujuGhw s1msuqN_whwS9YoueYgFF2ztJfyAcRF7RWBGWPSwwu 1oL1_wk4VLM8r8KtsYPcOTBD6-lj4fTJ2gR4eclrc9 12P2_2zJXsdsZAswWcHpudZFqzv2tw QGKDm_KjazTV\&sig=AHIEtbQ6zE7 tlGXmNklbVPsq-9bca1cVeQ\&pli=1

12. http://elcomercio.pe/tag/152778/metropolitano-de-lima 